\title{
Respiratory Inductance Plethysmography Improved Diagnostic Sensitivity and Specificity of Obstructive Sleep Apnea
}

\author{
Dmitriy Kogan MD, Arad Jain, Shawn Kimbro RPSGT, Guillermo Gutierrez MD PhD, and \\ Vivek Jain MD
}

\begin{abstract}
BACKGROUND: Respiratory inductance plethysmography (RIP) is a tool used during a polysomnogram (PSG), which serves as a surrogate of respiratory effort and can help detect inspiratory air-flow limitation. We hypothesize that RIP can improve the sensitivity and specificity of scoring hypopneas when compared with both the recommended and acceptable criteria of the American Academy of Sleep Medicine. METHODS: We retrospectively analyzed a cohort of 12 subjects who had no obstructive sleep apnea (OSA) or mild OSA on PSG when scored by the American Academy of Sleep Medicine acceptable criteria for hypopneas but had high clinical suspicion for a diagnosis of OSA. These subjects were rescored using the American Academy of Sleep Medicine recommended criteria as well as RIP. Hypopnea was scored when there was a 50\% decrease in the amplitude of the RIP sum channel (which combined input from chest and abdominal belts). OSA was diagnosed if the subjects had $>5$ respiratory events/h of sleep. The subject's response to CPAP was assessed by using a short questionnaire called the post-PSG sleep assessment. which evaluated subjective sleep quality. A positive response was considered an improvement in the post-PSG sleep assessment score after CPAP use. RESULTS: When scored using the American Academy of Sleep Medicine acceptable criteria, 10 subjects had a negative study, and 2 subjects had mild OSA for a sensitivity of $11 \%$ and specificity of $50 \%$. When scored using the recommended criteria, 10 subjects had OSA, and 2 were negative, for a sensitivity of $78 \%$ and specificity of $70 \%$. By RIP scoring, all 12 subjects had $>5$ respiratory events/h for a sensitivity of $100 \%$ and specificity of $\mathbf{7 5 \%}$. CONCLUSIONS: This small retrospective pilot study showed improved sensitivity and specificity when scoring hypopneas by RIP sum channel. Key words: polysomnography, respiratory inductance plethysmography, obstructive sleep apnea [Respir Care 2016;61(8):1033-1037. ( 2016 Daedalus Enterprises]
\end{abstract}

\section{Introduction}

Obstructive sleep apnea (OSA) is an extremely common disease in the United States and other industrialized countries, with reported rates of $10-20 \%$ in middle age men. ${ }^{1,2}$ Untreated OSA is associated with increased risk of coronary artery disease, cerebrovascular acci-

\footnotetext{
Dr Kogan is affiliated with the Division of Pulmonary and Critical Care Medicine, Medical College of Wisconsin, Milwaukee, Wisconsin. Mr Jain is affiliated with the Thomas Jefferson High School of Science and Technology, Alexandria, Virginia. Mr Kimbro, Dr Gutierrez, and Dr Jain are affiliated with the Division of Pulmonary and Critical Care Medicine, George Washington University, Washington, DC.
}

The authors have disclosed no conflicts of interest. dents, uncontrolled diabetes, and other complications. ${ }^{3-6}$ Individuals suffering from OSA are less productive and have increased health-care utilization and a higher risk of motor vehicle accidents. ${ }^{7,8}$ Despite the profound impact on personal health and detriment to society in general, OSA remains a widely underdiagnosed disease. Finkel et $\mathrm{al}^{9}$ found that $82 \%$ of adult surgical subjects in a single academic medical center had undiagnosed OSA.

Correspondence: Dmitriy Kogan MD, Division of Pulmonary and Critical Care Medicine, Medical College of Wisconsin, 9200 W. Wisconsin Ave. CLCC 5100, Milwaukee, WI 53226. E-mail: dkogan@mcw.edu.

DOI: $10.4187 /$ respcare. 04436 


\section{RIP IMPROVED DiAgNOSIS OF OSA}

The guidelines for diagnosing OSA on a polysomnogram (PSG) have been established and outlined by the American Academy of Sleep Medicine. ${ }^{10}$ In adults, an apnea is scored when there is a $\geq 90 \%$ decrease in peak signal excursion of pre-event baseline using an oronasal thermal sensor, positive airway pressure device flow, or an alternative apnea sensor, lasting for longer than $10 \mathrm{~s}$. Hypopnea is scored when the peak signal excursions decreases by $\geq 30 \%$ of pre-event baseline using a nasal pressure transducer, a positive airway pressure device flow, or an alternative sensor for $\geq 10 \mathrm{~s}$ in association with either $\geq 3 \%$ arterial oxygen desaturation or an arousal. This rule for scoring hypopnea is classified as a recommended criteria by the American Academy of Sleep Medicine..$^{10}$ The acceptable criteria for scoring hypopnea includes a signal excursion decrease of $\geq 30 \%$ of pre-event baseline, duration of $\geq 10 \mathrm{~s}$, and $\geq 4 \%$ desaturation from pre-event baseline. ${ }^{10}$ The authors of the scoring manual ${ }^{10}$ recommend that respiratory inductance plethysmography (RIP) be used for identification of apneas only when the oronasal thermal air-flow sensor is not functioning or the signal is not reliable. Similarly, RIP should only be used for scoring hypopneas when the nasal pressure transducer is not functioning or the signal is not reliable. ${ }^{11}$ Since the scoring manual ${ }^{10}$ does not endorse RIP as the first line diagnostic tool in the identification of respiratory events, it is not used universally in sleep centers.

RIP is a diagnostic technique used during polysomnography to evaluate patients' respiratory effort on a continuous basis. RIP consists of a driver module that generates an oscillating electrical current that travels through a wire inside belts placed around the subject's chest and abdomen. The electrical current generates a magnetic field that is modified by movement due to breathing. The signal is returned to the driver module and changed to analog format. The output displayed on the recording system is a representation of the patients' respiratory effort. ${ }^{11} \mathrm{~A}$ decrease in amplitude of the combined input from the chest and abdominal belts indicates a respiratory disturbance.

We hypothesized that RIP can improve the sensitivity and specificity of scoring hypopneas when compared with both the recommended ( $3 \%$ oxygen desaturation or arousal) and acceptable (4\% oxygen desaturation) criteria of the American Academy of Sleep Medicine. Furthermore, patients with clinical features of OSA, but with a non-diagnostic PSG based on the recommended and acceptable criteria, may benefit from CPAP if sleep-disordered breathing is indicated by RIP.

\section{Methods}

We studied a cohort of 12 subjects (Table 1) who had undergone PSG at the George Washington University Medical Faculty Associates Center for Sleep Disorders. Subjects who had no OSA or had mild OSA as scored

\section{QUICK LOOK}

\section{Current knowledge}

Respiratory inductance plethysmography (RIP) is a diagnostic technique used during a polysomnogram that serves as a surrogate of respiratory effort and can help detect air flow limitation. According to the scoring manual published by the American Academy of Sleep Medicine, RIP should only be used for scoring respiratory events when traditional air flow sensors are not functioning properly.

\section{What this paper contributes to our knowledge}

In a small retrospective pilot study, RIP showed improved sensitivity and specificity for scoring hypopneas compared with American Academy of Sleep Medicine recommended and acceptable criteria. Subjects who were found to have sleep-disordered breathing via RIP but not through traditional scoring techniques derived symptomatic benefit from positive airway pressure therapy.

per Berry et al ${ }^{10}$ but had high clinical suspicion for the diagnosis of OSA were included in the study. Subjects with either low clinical suspicion of OSA or an apneahypopnea index $\geq 15$ based on acceptable or recommended criteria were excluded from this study cohort. This cohort included 7 men and 5 women. Age range was 32-60 y with a mean age of $39.8 \mathrm{y}$. All subjects were either overweight or obese with mean body mass index of $33.36 \mathrm{~kg} / \mathrm{m}^{2}$. This research study was approved by the institutional review board of the George Washington University. Subjects were initially screened in the clinic by a sleep physician at our academic medical center and referred for polysomnography, given a high clinical suspicion for OSA, based on history and physical exam. Alice 5 software (Philips Respironics, Murrysville, Pennsylvania) was used to conduct the PSG. A nasal thermistor and nasal pressure transducer were used to evaluate air flow during the study. Respironics Z-RIP belts were used to evaluate respiratory effort. Initial scoring was conducted by a registered polysomnographic technologist using the American Academy of Sleep Medicine acceptable criteria in accordance with Medicare guidelines ${ }^{13}$ to score hypopneas. A clinical diagnosis of OSA was made when the apnea-hypopnea index was $\geq 5$ events/h of sleep.

Studies were rescored by the senior registered polysomnographic technologist, who was blinded to the original scoring. This subsequent scoring included the American Academy of Sleep Medicine recommended criteria for scoring hypopneas as well as an evaluation of the 


\section{RIP IMPROVED DIAGNOSIS OF OSA}

Table 1. Subject Demographics

\begin{tabular}{lclcc}
\hline \hline Subject & Age* & Sex & BMI, $\dagger \mathrm{kg} / \mathrm{m}^{2}$ & Epworth Score \\
\hline 1 & 32 & Male & 26.82 & 19 \\
2 & 42 & Male & 25.58 & 4 \\
3 & 47 & Male & 30.8 & 15 \\
4 & 46 & Male & 32.74 & 15 \\
5 & 32 & Male & 39.48 & 2 \\
6 & 45 & Female & 38.27 & 15 \\
7 & 60 & Female & 31.54 & 2 \\
8 & 28 & Male & 38.5 & 7 \\
9 & 33 & Female & 40.77 & 20 \\
10 & 54 & Female & 41.15 & 10 \\
11 & 58 & Male & 26.9 & 9 \\
12 & 47 & Female & 27.8 & 8 \\
\multicolumn{7}{l}{ * Mean age $=39.8$ y old. } & & & \\
$\dagger$ Mean body mass index $=33.36 \mathrm{~kg} / \mathrm{m}^{2}$. & & \\
\multicolumn{2}{l}{ body mass index } & & & \\
\hline
\end{tabular}

Table 2. Post-Polysomnogram Sleep Assessment

1. I feel more refreshed than usual this morning.

2. My quality of sleep last night was better than usual.

3. I slept deeper than usual last night.

4. I woke up fewer times than usual last night.

5. I slept longer than usual last night.

6. I feel better overall than usual this morning.

Responses were scored from 1 to 5 as follows: $1=$ strongly disagree, $2=$ slightly disagree,

$3=$ neither agree nor disagree, $4=$ slightly agree, $5=$ strongly agree

RIP sum channel. This channel combines input from chest and abdominal belts. A respiratory event was scored when there was a $\geq 50 \%$ decrease in the amplitude of the RIP sum channel curve. OSA was diagnosed if the subject had $\geq 5$ respiratory events/h of sleep. These subjects returned for a second overnight study with CPAP titration. After each night in the sleep center, subjects completed a post-PSG sleep assessment (Table 2), which is a short questionnaire that evaluated their subjective sleep quality. A subject's response to CPAP was assessed by comparing pre- and post-CPAP titration postPSG sleep assessment scores. A positive response after CPAP use was considered an improvement in post-PSG sleep assessment score and considered a surrogate for a true positive diagnostic study.

\section{Results}

When scored using the American Academy of Sleep Medicine acceptable criteria (4\% desaturation to score hypopnea), 10 subjects had a negative study, and 2 subjects had mild OSA (Table 3). When scored using the American
Table 3. Respiratory Events

\begin{tabular}{lccc}
\hline \hline \multirow{2}{*}{ Subject } & \multicolumn{2}{c}{ AASM Criteria } & \\
\cline { 2 - 3 } & Acceptable & Recommended & \\
\hline 1 & 0.9 & 15.7 & 18.1 \\
2 & 0.3 & 3.3 & 27.6 \\
3 & 7.6 & 16.8 & 42.5 \\
4 & 1.9 & 21.7 & 22.9 \\
5 & 0.2 & 12.2 & 16.2 \\
6 & 0.2 & 4.7 & 10.2 \\
7 & 3.2 & 17.4 & 26.9 \\
8 & 0 & 13.5 & 15.2 \\
9 & 2.1 & 10.2 & 14 \\
10 & 6.4 & 19.4 & 27.6 \\
11 & 1.9 & 6.6 & 19.3 \\
12 & 0.2 & 19.7 & 24.2 \\
& \multicolumn{3}{l}{} \\
\hline Respiratory events/h of sleep were scored by the American Academy of Sleep Medicine \\
acceptable and recommended criteria as well as respiratory inductance plethysmography. A \\
score of $\geq 5$ events/h was considered diagnostic for obstructive sleep apnea. \\
AASM = American Academy of Sleep Medicine \\
RIP = respiratory inductance plethysmography \\
\hline
\end{tabular}

Academy of Sleep Medicine recommended criteria (3\% desaturation or arousal for scoring hypopnea), 2 subjects still had a negative study, but the remaining 10 subjects had mild to moderate OSA. When scored using the RIP sum criteria, all 12 subjects had $>5$ respiratory events/h of sleep.

Sensitivities and specificities for each of the 3 criteria were then calculated. An increase in the post-PSG sleep assessment score after a night with CPAP was considered a true positive study (Table 4). This yielded a sensitivity of $11 \%$ and specificity of $50 \%$ when using the acceptable criteria, a sensitivity of $78 \%$ and specificity of $70 \%$ when scored using the recommended criteria, and a sensitivity of $100 \%$ and specificity of $75 \%$ when scored using the RIP sum criteria (Table 5).

\section{Discussion}

In-laboratory PSG is considered the accepted standard for diagnosing OSA; however, the cut-off apnea-hypopnea index values for mild $(\geq 5)$, moderate $(\geq 15)$, and severe OSA $(\geq 30)$ have been set arbitrarily and have not been shown to correlate with clinical features, such as somnolence and cognitive impairment. ${ }^{12,14}$ Our results show that RIP has improved sensitivity and specificity for scoring respiratory events compared with the recommended and acceptable American Academy of Sleep Medicine criteria. To our knowledge, this is the first study that directly compares these diagnostic methods. Air-flow obstruction can be detected by RIP due to a phase shift in thoracic and abdominal expansion (Fig. 1). During normal breathing, there is synchronous expansion of the chest and abdomen. 
Table 4. Post-Polysomnogram Sleep Assessment Scores

\begin{tabular}{lccc}
\hline \hline Subject & PPSA Pre & PPSA Post & PPSA Sum \\
\hline 1 & 17 & 23 & 6 \\
2 & 13 & 20 & 7 \\
3 & 18 & 27 & 9 \\
4 & 6 & 16 & 10 \\
5 & 12 & 24 & 12 \\
6 & 18 & 26 & 8 \\
7 & 26 & 28 & 2 \\
8 & 16 & 22 & 6 \\
9 & 22 & 17 & -5 \\
10 & 19 & 17 & -2 \\
11 & 10 & 8 & -2 \\
12 & 6 & 19 & 13
\end{tabular}

The post-polysomnogram sleep assessment was conducted after the initial polysomnogram and after CPAP titration to evaluate subjective sleep quality before and after CPAP use. A positive number for the post-polysomnogram sleep assessment summation indicates subjective improvement in sleep with CPAP.

PPSA pre $=$ post-polysomnogram sleep assessment conducted after the initial polysomnogram PPSA post $=$ post-polysomnogram sleep assessment conducted after CPAP titration

PPSA sum $=$ post-polysomnogram sleep assessment summation

Table 5. Sensitivity and Specificity of Diagnosing Obstructive Sleep Apnea by American Academy of Sleep Medicine Acceptable and Recommended Criteria as Well as Respiratory Inductance Plethysmography in This Cohort

\begin{tabular}{lcc}
\hline \hline Diagnostic Method & Sensitivity (\%) & Specificity (\%) \\
\hline Diagnostic criteria & & \\
Recommended & 78 & 70 \\
Acceptable & 11 & 50 \\
RIP & 100 & 75 \\
& & \\
RIP = respiratory inductance plethysmography & \\
\hline
\end{tabular}

Airway obstruction would create asynchronous respiratory effort, which would be seen as a phase shift in signals from chest and abdominal plethysmography belts. There would be a decrease in amplitude in the sum channel signal. Ultimately, an obstruction could cause a complete phase shift in thoracic and abdominal movements, which would result in paradoxical breathing. In this situation, signal flattening would be seen on the sum channel. The pathophysiology of respiratory obstruction in OSA makes RIP an ideal diagnostic tool.

Several studies have looked at the utility of RIP in diagnosing subjects with respiratory obstruction during sleep. Cantineau et $\mathrm{al}^{15}$ evaluated the accuracy of RIP during wakefulness and sleep in obese subjects with OSA. In that study, there was a mean error of $5.2 \%$ when minute ventilation was measured by RIP compared with integrated air-flow pneumotachography. It was concluded that quantitative measurement of ventilation by RIP is clinically useful in obese subjects with OSA. The authors suggested

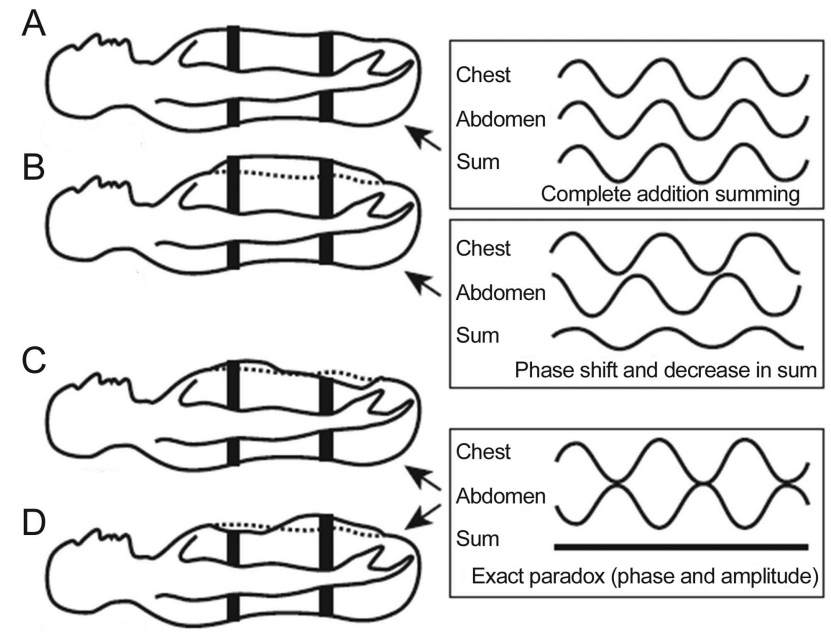

Fig. 1. Normal breathing (A), phase shifting (B), and paradoxical breathing ( $C$ and $D)$ as correlated to analog signal by respiratory inductance plethysmography. Courtesy Philips Respironics.

that it is appropriate to score hypopneas when a $50 \%$ decrease is seen in tidal volume assessed by RIP. We followed this recommendation when scoring hypopneas by RIP in our study.

Loube et $\mathrm{al}^{16}$ evaluated the accuracy of RIP in the detection of respiratory event-related arousals and diagnosis of upper-airway resistance syndrome as compared with the accepted standard of esophageal pressure measurement. The authors measured air flow via RIP by calculating the ratio of peak inspiratory flow to mean inspiratory flow. RIP-derived flow waveform flattening would occur as the ratio would approach 1.0 and would indicate air-flow obstruction. This study showed that there was $100 \%$ sensitivity and specificity for diagnosing upper-airway resistance syndrome when the ratio of peak inspiratory flow to mean inspiratory flow measurements were performed immediately before arousal. Both sensitivity and specificity values for diagnosis of upper-airway resistance syndrome were lower (67 and 80\%, respectively) when measurements were made randomly in stage 2 sleep. These results suggest that electroencephalography arousals should be taken into account when evaluating air-flow obstruction by RIP. We have not used this method in our study because we wanted to differentiate respiratory events diagnosed via RIP from the American Academy of Sleep Medicine recommended criteria.

Certain populations could especially benefit from improved diagnostic capabilities of RIP. Guilleminault et al ${ }^{17}$ described a cohort of women with symptoms of increased daytime somnolence who did not fit the conventional clinical picture of sleep-disordered breathing due to low body mass index and small neck circumference. These subjects had a respiratory disturbance index of $<5$ by traditional PSG and were mistakenly diagnosed as having chronic 


\section{RIP IMPROVED DiAgNOSIS OF OSA}

fatigue syndrome. It was only through esophageal pressure monitoring that the authors were able to make the diagnosis of upper-airway resistance syndrome. As described above, RIP has been shown to correlate with esophageal pressure monitoring in making the diagnosis of upperairway resistance syndrome.

An important limitation in our study is the lack of longterm follow-up of these subjects receiving CPAP. A positive response to treatment is based on subjective data from one night receiving CPAP. Long-term follow-up data with both subjective and objective outcome measures would certainly strengthen our argument. We did subsequently review the charts of the subjects to assess their subjective feelings about the response to CPAP, and all 12 subjects noted subjective improvements in their symptoms. The intention of our initial pilot study is to show a cohort of subjects with high clinical suspicion of OSA in whom the American Academy of Sleep Medicine standard diagnostic criteria are of limited value, whereas the use of RIP during the overnight PSG provides different and clinically relevant results.

\section{Conclusions}

This small retrospective pilot study showed improved sensitivity and specificity when scoring hypopneas by RIP sum channel. Further investigations are needed to determine whether RIP should be routinely used by sleep centers to score respiratory events in addition to the scoring per standard American Academy of Sleep Medicine diagnostic criteria.

\section{REFERENCES}

1. Young T, Palta M, Dempsey J, Skatrud J, Weber S, Badr S. The occurrence of sleep-disordered breathing among middle-aged adults. N Engl J Med 1993;328(17):1230-1235.

2. Kripke DF, Ancoli-Israel S, Klauber MR, Wingard DL, Mason WJ, Mullaney DJ. Prevalence of sleep-disordered breathing in ages 40-64 years: a population-based survey. Sleep 1997;20(1):65-76.

3. Schäfer H, Koehler U, Ewig S, Hasper E, Tasci S, Lüderitz B. Obstructive sleep apnea as a risk marker in coronary artery disease. Cardiology 1999;92(2):79-84.
4. Mooe T, Rabben T, Wiklund U, Franklin KA, Eriksson P. Sleepdisordered breathing in men with coronary artery disease. Chest 1996;109(3):659-663.

5. Mehra R, Benjamin EJ, Shahar E, Gottlieb DJ, Nawabit R, Kirchner $\mathrm{HL}$, et al. Association of nocturnal arrhythmias with sleep-disordered breathing: the Sleep Heart Health Study. Am J Respir Crit Care Med 2006;173(8):910-916.

6. Ismail K, Roberts K, Manning P, Manley C, Hill NS. OSA and pulmonary hypertension: time for a new look. Chest 2015;147(3): 847-861.

7. Colten HR, Altevogt BM. Sleep disorders and sleep deprivation: an unmet public health problem. Washington, DC: National Academies Press; 2006: 137-172.

8. George CF, Nickerson PK, Hanly PJ, Millar TW, Kryger MH. Sleep apnoea patients have more automobile accidents. Lancet 1987; 2(8556):447. Erratum in: Lancet 1987;2(8557):524.

9. Finkel KJ, Searleman AC, Tymkew H, Tanaka CY, Saager L, SaferZadeh E, Bottros M, Selvidge JA, Jacobsohn E, Pulley D, Duntley S, Becker C, Avidan MS. Prevalence of undiagnosed obstructive sleep apnea among adult surgical patients in an academic medical center. Sleep Med 2009;10(7):753-738.

10. Berry RB, Brooks R, Gamaldo CE, Harding SM, Lloyd RM, Marcus CL, Vaughn BV for the American Academy of Sleep Medicine. The AASM manual for the scoring of sleep and associated events: rules, terminology and technical specifications, version 2.0.2. http://www.aasmnet.org/scoringmanual/default.aspx. Darien, Illinois: American Academy of Sleep Medicine, 2013.

11. Sleep Diagnostic Equipment: an introduction for the sleep technologist. http://www.sleepdx.respironics.com/PDF/SleepDxEqMonograph. pdf. pp 20-27. Philips Respironics, 2012.

12. Redline S, Budhiraja R, Kapur V, Marcus CL, Mateika JH, Mehra R, et al. The scoring of respiratory events in sleep: reliability and validity. J Clin Sleep Med 2007;3(2):169-200.

13. Tunis S, Shuren J, Spencer FC, Chin J, Sheridan J. Decision memo for continuous positive airway pressure (CPAP) therapy for obstructive sleep apnea. https://www.cms.gov/medicare-coverage-database/ details/nca-decision-memo.aspx ?NCAId $=19 \&$ fromdb $=$ true. 2001.

14. Kingshott RN, Vennelle M, Hoy CJ, Engleman HM, Deary IJ, Douglas NJ. Predictors of improvements in daytime function outcomes with CPAP therapy. Am J Respir Crit Care Med 2000;161(3 Pt 1):866-871

15. Cantineau JP, Escourrou P, Sartene R, Gaultier C, Goldman M. Accuracy of respiratory inductive plethysmography during wakefulness and sleep in patients with obstructive sleep apnea. Chest 1992; 102(4):1145-1151.

16. Loube DI, Andrada T, Howard RS. Accuracy of respiratory inductive plethysmography for the diagnosis of upper airway resistance syndrome. Chest 1999;115(5):1333-1337.

17. Guilleminault C, Stoohs R, Kim YD, Chervin R, Black J, Clerk A. Upper airway sleep-disordered breathing in women. Ann Intern Med 1995;122(7):493-501.

This article is approved for Continuing Respiratory Care Education credit. For information and to obtain your CRCE

(free to AARC members) visit www.rcjournal.com

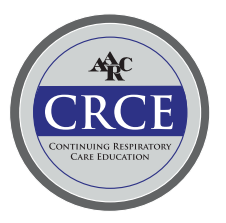

